\title{
Frontières
}

\section{Éros et Thanatos}

\section{Le dieu aux deux visages}

\section{Françoise Roy}

Volume 18, numéro 1, automne 2005

Hélas, célébrer la mort!

URI : https://id.erudit.org/iderudit/1074318ar

DOI : https://doi.org/10.7202/1074318ar

Aller au sommaire du numéro

Éditeur(s)

Université du Québec à Montréal

ISSN

1180-3479 (imprimé)

1916-0976 (numérique)

Découvrir la revue

Citer ce document

Roy, F. (2005). Éros et Thanatos : le dieu aux deux visages. Frontières, 18(1),

63-65. https://doi.org/10.7202/1074318ar

Ce document est protégé par la loi sur le droit d'auteur. L'utilisation des services d'Érudit (y compris la reproduction) est assujettie à sa politique d'utilisation que vous pouvez consulter en ligne.

https://apropos.erudit.org/fr/usagers/politique-dutilisation/
Cet article est diffusé et préservé par Érudit.

Érudit est un consortium interuniversitaire sans but lucratif composé de l’Université de Montréal, l'Université Laval et l'Université du Québec à Montréal. Il a pour mission la promotion et la valorisation de la recherche. https://www.erudit.org/fr/ 


\title{
ÉROS ET THANATOS LE DIEU AUX DEUX VISAGES
}

\author{
In memoriam, Susana Sanromán Ortiz ${ }^{1}$
}

\author{
Françoise Roy, \\ éditrice de la revue Tragaluz, Mexique.
}

Le mythe grec d'Hadès, seigneur des Enfers, est bien antérieur à la découverte, en 1930, de la dernière planète de notre système solaire portant son nom latinisé, Pluton. Sans pour autant chercher à justifier ou dénigrer les tenants de l'astrologie moderne qui s'est développée après la découverte astronomique des planètes trans-saturniennes, il est intéressant de constater quels symboles et archétypes ont été attribués à ce corps céleste que l'on a baptisé du nom romain du dieu de la mort, et qui signe l'appartenance astrologique à la constellation du Scorpion. Tout ce qui touche aux profondeurs tombe sous la maîtrise de cette déité; le mythe disait que nul ne pouvait regarder Hadès sous peine de mourir d'épouvante et qu'il portait un casque le rendant invisible, en analogie avec son goût pour les choses cachées, le secret, ce qui ne doit pas être vu.

Divinité liée à l'instinct, aux pulsions, aux obsessions, aux fantasmes et aux peurs archaïques, aux mutations et transmutations, aux transformations, aux processus de renaissance et régénération, à la magie et au pouvoir occulte, Pluton était déjà considéré au temps des Grecs comme une force maléfique. Il y a donc trois volets à la signification mythique de Pluton: tout ce qui est caché, tout ce qui a trait aux pouvoirs profonds, et la dyade naissancemort, cet inséparable duo que les Grecs ont assigné, bien à propos, à une seule et même divinité.

L'inconscient, les sciences occultes, le mystère, les tabous, les phobies, la subversion, la coercition, tout ce qui est tortueux et complexe, les secrets, la vie sexuelle, tout ce qui est tapi sous la surface en attendant d'être révélé ou exposé à la vue, relèvent du premier volet. La deuxième facette de Pluton englobe le pouvoir qui prend naissance dans le noir ou les enfers symboliques de l'existence: épuration, crime, compulsions, tout ce qui tient des pulsions viscérales, du magnétisme, des forces chtoniennes et des actes passionnels, dont l'enlèvement (il suffit de se souvenir du rapt de Perséphone par Hadès, l'événement clé du mythe plutonien), la destruction et le terrorisme. La psychologie moderne, sous l'égide de Freud, a donné à ce volet le nom de «ça», le moi étant lié au Soleil, et le surmoi à Saturne.

Mais dans la version du mythe la plus intimement liée au monde physique et aux lois mêmes de l'existence, on doit faire appel à l'association indissoluble entre la vie et la mort, le début et la fin: c'est alors que la naissance et son contraire, l'accouchement et l'enterrement, la rénovation, les processus de duplication (incluant les imprimantes et photocopieuses), la résurrection, la putréfaction (celle de la graine qui doit passer par ce processus vital qu'est la décomposition avant de pouvoir germer) et la fermentation sont régis, astrologiquement et mythologiquement parlant, par le souverain du royaume des morts. Le mois de novembre, devenus dans l'iconographie et le calendrier chrétien celui des défunts, correspond à l'échelle du zodiaque à la période de l'existence, végétale et par analogie humane, menacée par le danger de la chute. Les objets tombant sous la dominance de l'énergie plutonienne en disent long sur sa nature souterraine et explosive, qui fuit la lumière du jour: les cloaques et égouts, les bombes, les volcans, l'énergie atomique, les bactéries, les virus et le vide. Les assignations corporelles de l'énergie plutonienne sont aussi très révélatrices: le système reproducteur, les organes génitaux, le gros intestin (le dernier endroit où séjourne ce qui doit être éliminé par le corps), la glande pituitaire (le siège de l'âme, selon plusieurs grands courants ésotériques) et les excroissances (tumeurs, verrues, taches de naissance).

La dualité vie et mort, origine et eschatologie, est au centre du mythe plutonien. Si l'on célèbre la vie, la venue d'un enfant étant universellement un motif de festivité (et je pense ici à la scène magnifique qui ouvre le roman de Djuna Barnes, Le bois de la nuit, décrivant la célébration ayant lieu lors de la naissance d'un enfant juif), pourquoi ne pas célébrer la mort? Si autant le berceau que le tombeau sont des phénomènes mythiquement plutoniens, il faut peut-être faire référence aux racines étymologiques du mot Pluton pour y voir plus clair. Le vocable en soi vient de Ploutos, qui en grec signifie «richesses, trésor». D'ailleurs, Pluton était au départ dieu de la fécondité et de l'abondance des récoltes. Ce n'est que plus tard qu'il a acquis son visage de violeur, de maître de cérémonie de tout processus d'initiation, qui implique toujours une perte, un sacrifice, un renoncement douloureux. C'est dans les entrailles de la Terre, au plus profond de l'être, là où se trouvent les déchets, les choses qui doivent être cachées à la vue et enterrées, que gisent les plus grandes richesses; c'est cela que veut souligner le mythe. L'iconographie occidentale, le monde grec, sont riches en évocations de la renaissance qui doit forcément passer par la mort, soit-elle physique ou figurée. Aux racines de notre civilisation, nous avons l'oiseau phénix qui renaît de ses propres 


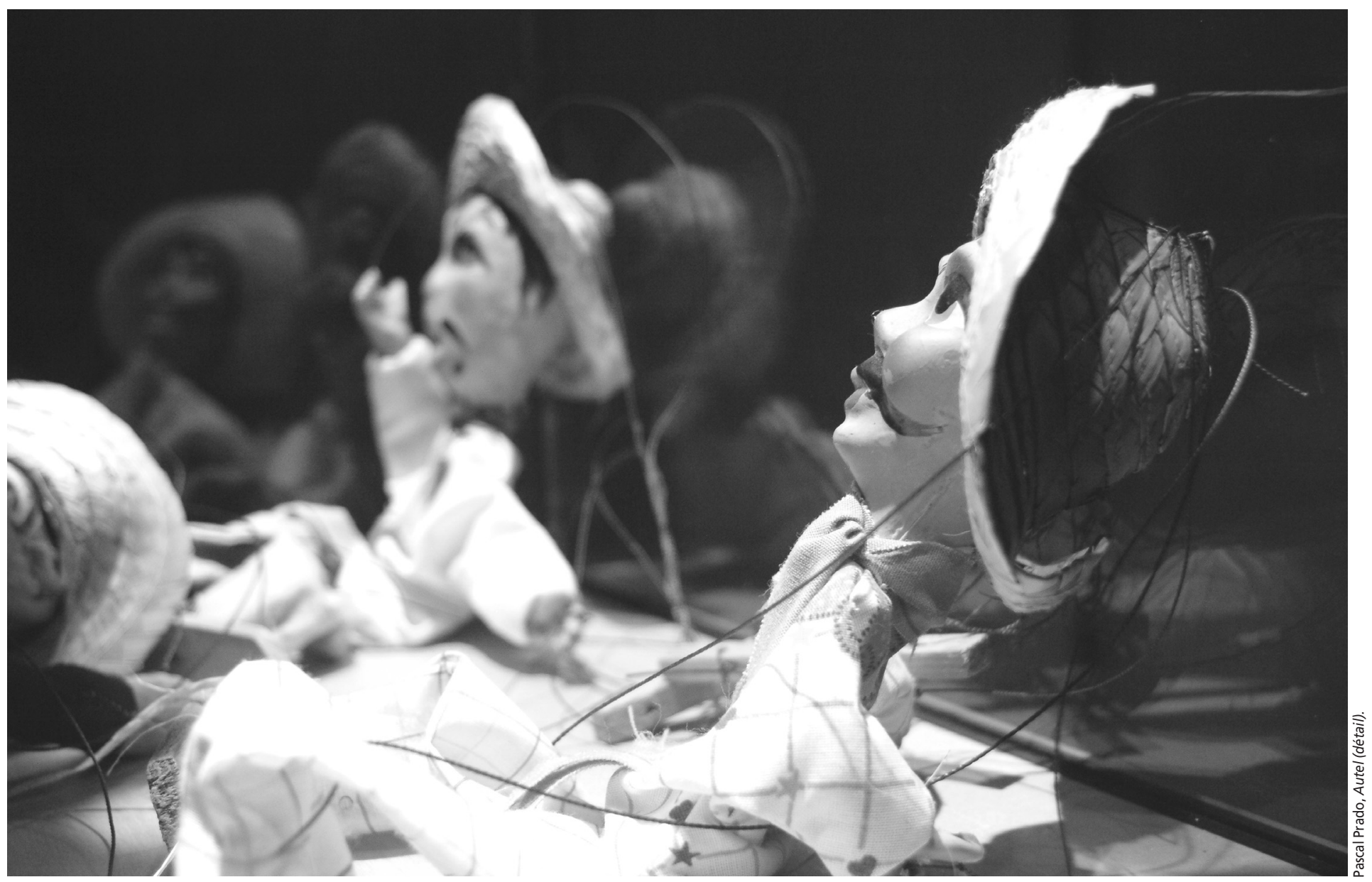

cendres. Nous avons aussi Orphée, qui doit descendre aux enfers pour y retrouver son âme perdue, et ne doit pas se retourner pour voir Eurydice, sous peine de la perdre à jamais. Les autres traditions mythiques à travers le monde, elles aussi, ont toutes une représentation du triomphe de la vie après un transit au royaume des ombres. La descente du Christ aux enfers, le mythe de Quetzalcoatl au Mexique précolombien, et j'en passe. Il n'est pas surprenant, étant donné la pensée analogique des anciens Grecs, qu'Éros et Thanatos, la pulsion de vie et la pulsion de mort, aient entretenu de tels liens de parenté qu'ils soient devenus les deux côtés d'une même monnaie, les deux visages de la même divinité, les deux pendants d'une même pulsion, selon la psychanalyse.

Mais qui donc est cet Éros? Les racines étymologiques du mot «amour» chez les Grecs illustrent parfaitement son identité. Nous savons que les Grecs avaient plusieurs mots pour désigner ce que nous, Occidentaux modernes, regroupons, très peu sagement d'ailleurs, sous un même nom: l'amour. Il y avait, suivant l'ordre du zodiaque, le concept d'epithemia, l'amour tel qu'il se manifeste sous le signe du Taureau. Il s'agit d'un amour passablement terrestre, très charnel, qui mise sur la jouissance cor- porelle, la beauté, le confort, la récompense des sens. Ensuite, suivant l'ordre de la croix des signes fixes, on retrouvait le concept de phylia, la version de l'amour vécu sous le signe du Lion. Voilà la liaison romantique dont la littérature universelle nous a légué la plus parfaite version archétypique avec l'histoire de Roméo et Juliette. Ce type d'amour, grandement idéalisé, qui tourne autour de la conquête, l'amant potentiel cherchant à étudier l'objet de son désir afin d'attirer son attention, est finalement lié à la question de l'orgueil, de l'amourpropre, et se termine souvent aussitôt qu'il est consumé.

Le dernier mot désignant l'amour (et je passe volontairement celui qui correspond au signe du Scorpion), agape, fait appel à l'autonomie et à la liberté des amants. Les Grecs désignaient par ce mot une liaison où la raison prime sur le cœur, où le bonheur des amants et le respect de leur individualité sont si importants qu'une rupture est préférable à l'établissement de conditions entravant le développement et l'épanouissement individuel. C'est entre epithemia et agape que se trouve l'amour que les Grecs avaient baptisé du nom d'eros. Il s'agit d'un amour passionnel, possessif, coloré par le concept du «tout ou rien » attribué au signe du Scorpion, et donc au dieu qui le représente le plus fidèlement, Hadès / Pluton. Mais la condition fondamentale de ce type d'amour, plus encore que la possession et l'exclusivité ou même l'intensité des sentiments, est la métamorphose. Dans la relation dominée par le concept d'eros, il y a transformation mutuelle des amants au moment de se livrer l'un à l'autre; la vie s'épanouit, la richesse des profondeurs monte à la surface, et symboliquement, les membres du couple doivent mettre à mort leur individualité, mourir à leur ancienne identité pour effectuer la fusion tant émotive que sexuelle. N'est-ce pas cela que mourir, laisser son corps pour s'ouvrir à une nouvelle identité où il y a transmutation totale de l'énergie?

Voilà pourquoi pour les Grecs, le dieu qui préside sur le royaume des morts est à la fois celui de la vie, de la sexualité, de l'intimité, des révolutions intérieures et du cycle vital: gestation, naissance, croissance et trépas. S'il y a un endroit physique qui symbolise Pluton, c'est bien l'alcôve, la chambre close, dont le cercueil, le tombeau, sont des versions étiolées. Rappelonsnous que, selon l'orphisme, Éros, dans la première version du mythe, était né de l'œuf cosmique engendré par la nuit. Cet œuf primordial contenait en lui le germe de toute manifestation et la loi de toute renaissance. À la naissance d'Éros, l'œuf 
se brisait en deux moitiés qui ensuite formaient la Terre (Gaïa) et le Ciel (Ouranos), dont la hiérogamie engendrait à elle seule tous les êtres. La deuxième version, plus connue, fait d'Éros le fils d'Aphrodite, qui séduit la belle Psyché. Mais pour échapper à la jalousie maternelle, Éros exile sa bienaimée dans un palais où il ne peut lui rendre visite que la nuit, et ce, dans la noirceur totale. Cependant, Psyché, aiguillonnée par la curiosité et le désir, viole la prohibition, et un soir, ose regarder le visage de son bien-aimé à la lumière d'une lampe à l'huile. Éblouie par la beauté d'Éros, elle s'incline, l'huile de la lampe tombant sur son amant, qui se réveille alors et l'abandonne à jamais, pour éviter que ne s'abatte sur eux la malédiction maternelle. C'est alors que Psyché doit faire face à Thanatos, la perte totale, le bouleversement, la nuit obscure de l'âme: personnification de la mort, Thanatos était le fils de la nuit et le frère du rêve, un bel éphèbe ailé dont les attributs étaient le pavot et une torche éteinte. Le rêve, l'évanouissement, le flou, les effets stupéfiants de la fleur opiacée, tous sont des symboles partagés tant par l'amour que la mort. Il y a le repos, et en même temps l'intensité, comme les deux côtés d'une monnaie. D'ailleurs, cette liaison passionnelle entre Éros et Thanatos, Marguerite Yourcenar ne l'exprime-t-elle pas de façon magistrale dans le roman qui devint son chef-d'œuvre, lorsque l'empereur Hadrien avoue qu'il ne savait pas alors que la mort pouvait devenir l'objet d'une ardeur aveugle, d'une avidité semblable à l'amour?

En ce sens, la mort est motif de célébration: on doit la pleurer et l'accueillir en même temps, car elle participe du même archétype que les processus érotiques, les passions totalisantes, la renaissance qui suit la crise, le sommeil que représentent le pavot et la torche éteinte. Le lieu où l'on doit passer pour accéder à cette transmutation est certes baigné dans la grande noirceur. C'est le monde souterrain, celui des égouts, des secrets. Dans la nature, suivant le cycle des moissons, cela correspond à la pourriture de la graine enterrée (voilà encore le symbolisme plutonien relatif aux choses enfouies, qui s'incarne à merveille dans le mythe d'Éros et Psyché), prisonnière dans la froide obscurité du sol avant de pouvoir germer. Mais parler de transmutation implique qu'il y a transformation de la forme, de l'énergie, de l'état d'être, et non pas leur annulation ou leur anéantissement. J'estime donc que la seule condition pour célébrer la mort, parce qu'on croit accéder à une mutation signée par une transfiguration de la forme, comme le symbolise le mythe plutonien, est la croyance en une forme de survie à la mort physique. S'il y a absence d'un credo où l'on maintient la conviction d'une vie qui ne fait

LA MORT EST MOTIF DE CÉLÉBRATION:

ON DOIT LA PLEURER ET L'ACCUEILLIR EN MÊME TEMPS,

CAR ELLE PARTICIPE DU MÊME ARCHÉTYPE

QUE LES PROCESSUS ÉROTIQUES, LES PASSIONS TOTALISANTES,

LA RENAISSANCE QUI SUIT À LA CRISE.

que changer d'état de conscience, l'appellet-on métempsycose comme au temps des Grecs, réincarnation comme chez les Orientaux, vie éternelle comme le stipule le christianisme, on n'a aucune raison de célébrer la mort, si ce n'est comme d'une délivrance des vicissitudes de l'existence (fatigue, maladie, invalidité, douleur, souffrance physique ou morale). Le néant, le vide, la fin ultime de l'existence, telle qu'elle est proposée par les systèmes de pensée existentialistes n'envisageant pas de survie après la mort, ne sauraient être célébrés.

Que faire de ces beaux mythes où la force vitale, érotique, qui nourrit l'existence, l'alimente, l'enrichit et la conserve, doit rencontrer forcément Thanatos, la chute et ensuite l'apaisement, pour accomplir son destin, dans une société presque complètement sécularisée, où l'au-delà et ses récompenses (fussent-elles envisagées en terme de finalité, comme dans le monothéisme, ou d'évolution, comme chez les Égyptiens, les Grecs ou les Orientaux) sont devenues chimères? À mon sens, on ne peut célébrer la mort dans un contexte d'athéisme ou d'agnosticisme. Il n'y a que le concept de transformation qui puisse lui donner un sens, que le changement, que l'investiture radicale, qui au niveau symbolique sont au cœur du concept d'eros, l'amour tel qu'il est décrit dans la constellation du Scorpion. Ce qui est intéressant, c'est de voir que même à une époque où la plupart des gens meurent relativement isolés, dans la froideur d'un hôpital, souvent privés de rites car on ne croit plus et on improvise l'extrême-onction ou un type quelconque de bénédiction spirituelle "au cas où », on aurait gardé encore beaucoup de similitudes avec les mythes d'une civilisation (le berceau de la culture occidentale) où l'immortalité de l'âme était un aspect essentiel de la condition humaine. La mort reste encore une affaire cachée comme tout événement plutonien, confinée si possible aux chambres d'hôpitaux, aux mouroirs, gardant intacte toute la signification occulte de la huitième maison, qui dans le zodiaque est le secteur de vie régi par le dieu Pluton. Si la huitième maison est celle de la dépossession, des gains et des pertes, de l'altérité, on peut difficilement imaginer un événement où simultanément on gagne tout et l'on perd tout comme la mort physique. Mais ce «tout gagner» suppose l'existence d'une vie après la mort corporelle. Sinon, mourir, c'est n'accomplir que la moitié de la promesse plutonienne: «tout perdre », renoncer à sa corporalité pour ne tomber que dans le néant, sans subir pour autant de métamorphose. Pour les hommes de la prémodernité, qui ont vécu lorsque les dieux n'étaient pas encore disparus, la dépossession physique n'était qu'un pas vers la vie éternelle, et la huitième maison, fidèle à leur conception du monde, est vraiment la demeure où le gain succède à la perte. Soit dit en passant, les rites aussi sont un attribut de cette maison, et peu de circonstances sont entourées d'autant de rites, dans la majorité des sociétés et des époques, que les veillées funéraires et les enterrements. Les rituels de célébration n'auraient aucun sens si le trépas était une fin en soi et non pas l'acte de franchir un seuil au-delà duquel se trouve la renaissance spirituelle. Comme nous le confirme la philosophe espagnole María Zambrano, toute culture est finalement la concrétisation de l'espoir que nous avons de naître à nouveau.

\section{Bibliographie}

BARNES, D. (1986). Le bois de la nuit, Paris, Le Seuil.

BECKER, U. (1996). Enciclopedia de los símbolos, Mexico, Editorial Océano de México.

GUIRAND, F. (dir.) (1935). Mythologie générale, Paris, Librairie Larousse.

PHILIBERT, M. (1998). Dictionnaire des mythologies, Paris, Maxi-Livres-Profrance.

POCH-KADE, A. (1997). La casa ocho. Una dimensión sicológica de la astrología, Buenos Aires, Editorial Kier.

SASPORTAS, H (1990). Los dioses del cambio. El dolor, las crisis y los tránsitos de Urano, Neptuno y Plutón, Barcelone, Ediciones Urano.

YOURCENAR, M. (1951). Mémoires d'Hadrien, Paris, Gallimard.

\section{Note}

1. À mon amie Susana (1957-2005), décédée le 4 avril dernier, ses derniers jours écoulés dans l'éblouissante lumière printanière de Lagos de Moreno, au Mexique, après une lutte de 15 ans contre le lupus et l'insuffisance rénale, et dont le courage et la passion ont prévalu tant dans la vie que dans la mort. 